\title{
Hyperplastic-Like Mucosal Change in Crohn's Disease: An Unusual Form of Dysplasia?
}

\author{
Sony P. Kilgore, M.D., Jessica E. Sigel, M.D., John R. Goldblum, M.D. \\ Department of Anatomic Pathology, Cleveland Clinic Foundation, Cleveland, Ohio
}

Patients with Crohn's disease are at increased risk of developing intestinal adenocarcinoma. Dysplasia is both a marker and a precursor of adenocarcinoma in this setting. In a review of our cases of Crohn's-related adenocarcinoma, we noted a peculiar hyperplastic-like mucosal change (HPC) in mucosa both adjacent to and distant from the adenocarcinoma in some cases. However, the significance of this change is unknown.

We evaluated 30 cases of Crohn's-related adenocarcinoma and 30 age- and site-matched resection specimens with Crohn's disease without adenocarcinoma to determine the prevalence of this mucosal alteration in these groups. HPC was recognized by a diffuse expanse of flat mucosa with an architecture resembling that seen in colorectal hyperplastic polyps and composed of cells with cytologically bland basal nuclei and apical cytoplasmic mucin distention. The relationship of the HPC to the adenocarcinoma was noted in the Crohn's-related adenocarcinoma cases. An immunohistochemical stain for p53 (antibody D07) was performed on all cases with HPC in both groups.

HPC was identified in 10 of $30(33 \%)$ cases of Crohn's-related adenocarcinoma compared with 3 of $30(10 \%)$ cases in the control group $(P=.03)$. In the 10 cases of Crohn's-related adenocarcinoma with HPC, this alteration was found adjacent to the adenocarcinoma in 3 cases, distant to the adenocarcinoma in 5 cases, and both adjacent to and distal from the adenocarcinoma in 2 cases. In two specimens, HPC was seen immediately adjacent to adenocarcinoma in the absence of adjacent dysplasia. p53 immunoreactivity was noted in HPC in 5 of 10 (50\%) Crohn's-related adenocarcinomas. In contrast, p53 immunoreactivity was not seen in HPC in the three control cases with this mucosal alteration.

Copyright () 2000 by The United States and Canadian Academy of Pathology, Inc.

VOL. 13, NO. 7, P. 797, 2000 Printed in the U.S.A.

Date of acceptance: January 28, 2000.

Address reprint requests to: John R. Goldblum, M.D., Cleveland Clinic Foundation, 9500 Euclid Avenue L25, Cleveland, OH 44195; e-mail: goldblj@ccf.org; fax: 216-445-6967.
In conclusion, HPC is found significantly more commonly in mucosa both adjacent to and distant from Crohn's-related adenocarcinoma when compared with age- and site-matched controls. In addition, p53 immunoreactivity is more commonly seen in HPC in cases of Crohn's-related adenocarcinoma compared with controls. These data suggest that this mucosal alteration may, in some cases, represent an unusual form of dysplasia in this setting.

KEY WORDS: Crohn's disease, Dysplasia, Hyperplasia, p53.

Mod Pathol 2000;13(7):797-801

Patients with inflammatory bowel disease (IBD) are at increased risk for developing intestinal adenocarcinoma, although the risk seems to be greater in patients with ulcerative colitis than Crohn's disease (1-5). We recently reviewed specimens from a cohort of patients with Crohn's-related adenocarcinoma to evaluate the relationship between dysplasia and adenocarcinoma (6). During this evaluation, we noted a peculiar hyperplastic-like mucosal change (HPC) in mucosa both adjacent to and distant from the adenocarcinoma in some cases. The goal of this study was to determine the prevalence of this change in resection specimens for Crohn'srelated adenocarcinoma compared with a control group of age- and site-matched resection specimens with Crohn's disease not complicated by adenocarcinoma.

\section{MATERIALS AND METHODS}

We searched the files of the Department of Anatomic Pathology at the Cleveland Clinic Foundation for all cases of Crohn's-related adenocarcinoma from 1977 to 1997 . Cases were included in the study only if they were resection specimens and only if the adenocarcinoma arose in an area of bowel involved by Crohn's disease to minimize the evaluation of cases in which the diagnosis of adenocarcinoma and Crohn's disease was coincidental (sporadic adenocarcinoma). In addition, a control group of 30 age- and site-matched resection spec- 
imens with Crohn's disease not complicated by adenocarcinoma were studied. All available hematoxylin and eosin-stained slides were reviewed to determine the prevalence of HPC in these two groups. HPC was recognized by a diffuse expanse of flat mucosa with an architecture resembling that seen in colorectal hyperplastic polyps and composed of cells with cytologically bland basal nuclei and apical cytoplasmic mucin distention (Figs. 1$3)$. The relationship of the HPC to the adenocarcinoma was noted in the Crohn's-related adenocarcinoma cases. If HPC and adenocarcinoma were present in the same histologic section, then the HPC was considered to be adjacent to the carcinoma. In all other instances, the HPC was considered to be distant from the carcinoma. An immunohistochemical stain for p53 (antibody DO7) was performed on all cases with HPC in both groups, using a labeled streptavidin-biotin system by means of an automated immunostainer (Ventana 320; Ventana Medical Systems, Tucson, AZ). A case was considered to be p53 positive if more than $25 \%$ of the cells in the HPC area showed nuclear immunoreactivity with this antibody.

\section{RESULTS}

The cohort of patients with Crohn's-related adenocarcinoma included 21 men and 9 women with an age range from 18 to 84 years (mean age, 49 years). Eight tumors arose within the small bowel, 14 within the colon, and 8 within the rectum, including 2 cases in out-of-circuit rectums. Dysplasia was found adjacent to the carcinoma in 26 cases (87\%) and distant to the carcinoma in 10 cases (33\%). All foci of dysplasia arose in areas involved by Crohn's disease.

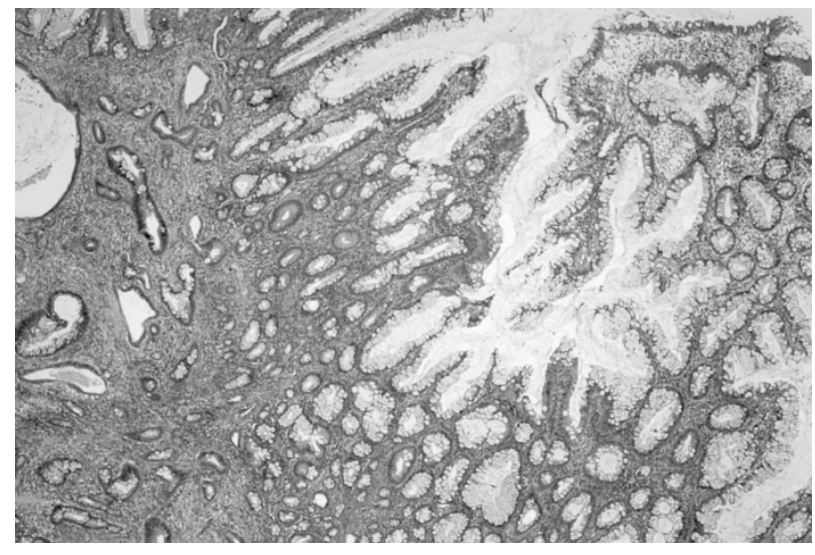

FIGURE 1. Low-magnification view of hyperplastic-like mucosal change seen adjacent to an invasive adenocarcinoma. Although there is some element of tangential sectioning, one can appreciate the relationship of the hyperplastic-like mucosal change to the underlying adenocarcinoma. This case lacked recognizable dysplasia adjacent to the invasive adenocarcinoma.

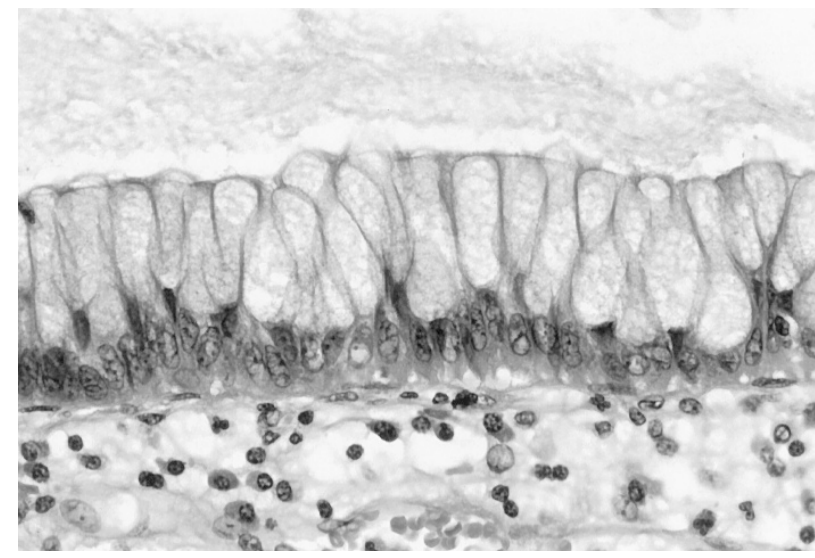

FIGURE 2. High-magnification view of mucin-filled cells in hyperplastic-like mucosal change.

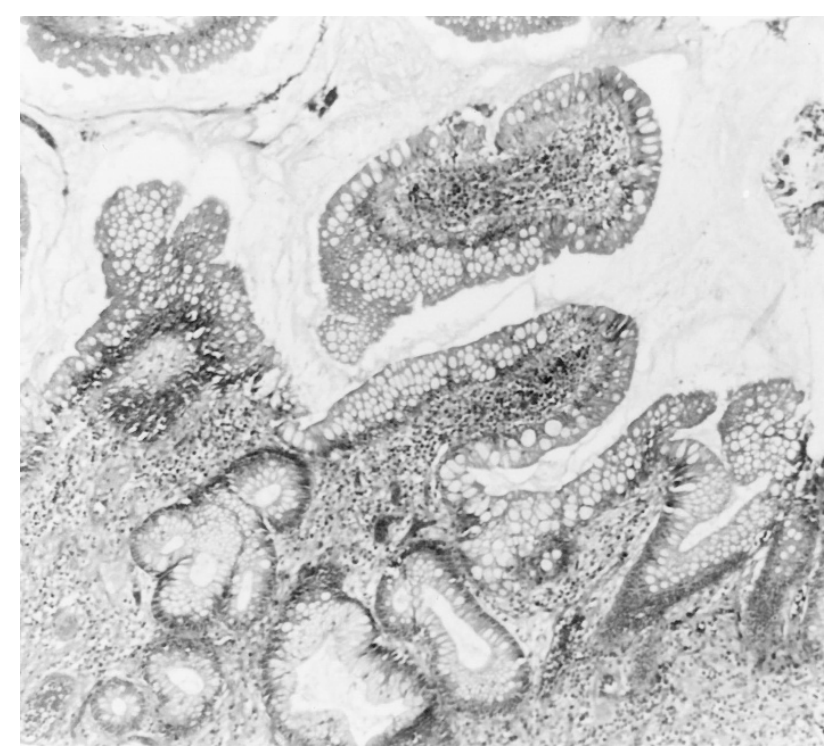

FIGURE 3. Hyperplastic-like mucosal change in a case of Crohn's disease that was not associated with adenocarcinoma.

HPC was identified in 10 cases (33\%) of Crohn'srelated adenocarcinoma, compared with only 3 cases $(10 \%)$ of resected Crohn's disease without adenocarcinoma $(P=.03)$ (Table 1$)$. The morphology of the HPC was similar in carcinoma and noncarcinoma-associated cases. This mucosal alteration was found distant from the carcinoma in five

TABLE 1. Prevalence of Hyperplastic-Like Mucosal Change in Crohn's Disease with and without Adenocarcinoma

\begin{tabular}{lcc}
\hline & $\begin{array}{c}\text { Present } \\
(\%)\end{array}$ & $\begin{array}{c}\text { Absent } \\
(\%)\end{array}$ \\
\hline Crohn's-related adenocarcinoma $(n=30)$ & $10(33 \%)^{a}$ & $20(67 \%)$ \\
Distant from adenocarcinoma & $5(17 \%)$ & \\
Adjacent to adenocarcinoma & $3(10 \%)$ & \\
$\quad \begin{array}{l}\text { Both adjacent to and distant from } \\
\quad \text { adenocarcinoma }\end{array}$ & $2(7 \%)$ & \\
Crohn's disease without adenocarcinoma & $3(10 \%)^{a}$ & $27(90 \%)$ \\
$\quad(n=30)$ & & \\
\hline$a p=.03$. & &
\end{tabular}


cases $(17 \%)$, adjacent to the carcinoma in three cases $(10 \%)$ and both adjacent to and distant from the carcinoma in two cases (7\%). In two specimens, HPC was seen adjacent to the carcinoma in the absence of adjacent dysplasia.

Ten cases with HPC in Crohn's-related adenocarcinoma were immunostained with an antibody to p53, including 6 HPC foci distant from carcinoma and 4 HPC foci adjacent to carcinoma (Table 2). Two of six HPC foci distant from carcinoma stained strongly for p53 (Fig. 4), whereas the surrounding non-HPC mucosa did not stain. Three of four HPC foci adjacent to carcinoma stained strongly for p53, but the surrounding non-HPC nondysplastic mucosa was p53 negative. Two of these four cases had recognizable adjacent dysplasia, both of which also stained for p53. Of the two cases with HPC adjacent to carcinoma without recognizable adjacent dysplasia, one case stained strongly for p53. In general, the p53 staining was stronger at the base of the HPC, with little staining of the surface epithelium (Fig. 5). All four adenocarcinomas that were found adjacent to the HPC stained strongly for p53, including the single case in which the HPC was p53 negative. In contrast, none of the three HPC foci in cases of Crohn's disease without adenocarcinoma stained for p53.

\section{DISCUSSION}

It has been well established that patients with Crohn's disease are at increased risk of developing intestinal adenocarcinoma (1-5). However, there is a relative paucity of data regarding a dysplasiacarcinoma sequence in Crohn's disease when compared with ulcerative colitis. We recently reported our experience with intestinal adenocarcinoma complicating Crohn's disease (6) and found that the vast majority of cases (87\%) had dysplasia adjacent to the carcinoma, but only $33 \%$ of cases had dysplasia found in mucosa distant from the carcinoma. During this evaluation, we noted a peculiar hyperplastic-like mucosal alteration in mucosa

TABLE 2. p53 Immunoreactivity in Hyperplastic-Like Mucosal Change in Crohn's Disease with and without Adenocarcinoma

\begin{tabular}{lcc}
\hline & p53 & p53 \\
Positive & Negative \\
\hline $\begin{array}{l}\text { Crohn's-related adenocarcinoma with HPC } \\
(n=10)\end{array}$ & 5 & 5 \\
HPC foci distant from adenocarcinoma $(n=6)$ & 2 & 4 \\
HPC foci adjacent to adenocarcinoma $(n=4)$ & 3 & 1 \\
HPC foci adjacent to adenocarcinoma without & 1 & 1 \\
$\quad$ adjacent dysplasia & & \\
Dysplasia adjacent to adenocarcinoma $(n=2)$ & 2 & 0 \\
Adenocarcinoma adjacent to HPC $(n=4)$ & 4 & 0 \\
Crohn's disease without adenocarcinoma with & 0 & 3 \\
$\quad$ HPC $(n=3)$ & & \\
\hline
\end{tabular}

HPC, hyperplastic-like mucosal change

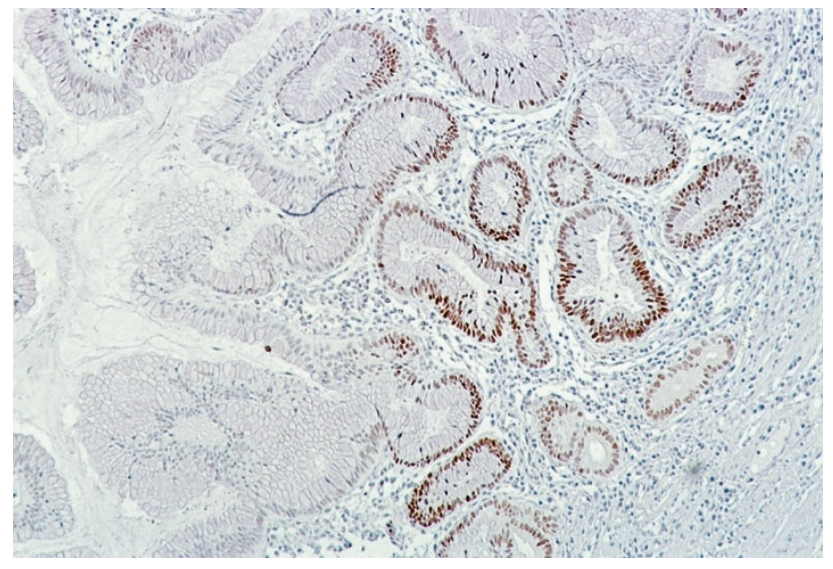

FIGURE 4. Low-magnification view of immunohistochemical stain for p53 in hyperplastic-like mucosa in a case of Crohn's-related adenocarcinoma. This focus was found distant from the adenocarcinoma. Most of the cells show strong nuclear immunoreactivity, particularly at the base.

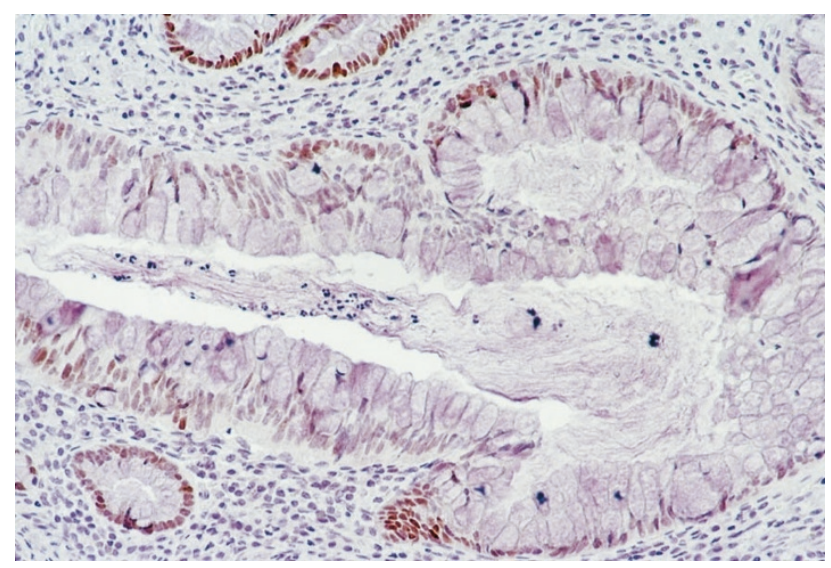

FIGURE 5. High-magnification view of nuclear immunoreactivity for p53 in hyperplastic-like mucosa found adjacent to a Crohn's-related adenocarcinoma. In this case, there was no recognizable dysplasia adjacent to the adenocarcinoma. The surrounding nonhyperplastic mucosa did not stain for p53, but the adjacent adenocarcinoma was strongly p53 positive.

both adjacent to and distant from the adenocarcinoma in a subset of these cases. However, the nature and significance of this mucosa is not known, and its existence has not been emphasized in the previous literature on Crohn's-related intestinal adenocarcinoma.

This mucosal alteration was found significantly more commonly in resected Crohn's disease associated with adenocarcinoma (33\%) when compared with cases of resected Crohn's disease without adenocarcinoma (10\%). Histologically, HPC closely resembles transitional mucosa, a term first coined by Filipe in 1969 (7) to describe nonpolypoid, nonneoplastic colorectal mucosa found adjacent to colorectal adenocarcinoma (8-10). Since its initial description, the histologic $(11,12)$, histochemical $(13,14)$, immunohistochemical $(15,16)$, and ultrastructural (17) features of this mucosa have been further delineated. Histologically, transitional mu- 
cosa is characterized by an increase in mucosal thickness with lengthening of the crypts and goblet cell hyperplasia with marked mucin distention of goblet cells (7). Histochemically, it is characterized by an increased sialomucin content of the goblet cells, which contrasts to the sulfomucin-rich mucosa of the normal colon $(13,14)$. Riddell and Levin (17) and others (8) also described characteristic ultrastructural alterations in transitional mucosa. Some authors suggested that this mucosa likely represents a preneoplastic alteration, given its unique histologic, histochemical, and ultrastructural features, as well as its identification adjacent to colorectal adenomas and carcinomas and other conditions associated with increased colorectal cancer risk $(18,19)$. Others, however, suggested that transitional mucosa represents a nonspecific secondary change because similar mucosal alterations may be seen adjacent to tumors other than adenocarcinoma $(13,14)$, as well as in mucosa adjacent to a heterogeneous group of colorectal diseases such as mucosal prolapse (20), diverticular disease (21), and endometriosis (22). Sawady et al. (16) found the immunohistochemical features of transitional mucosa adjacent to primary colorectal adenocarcinoma and metastases to the colon to be identical, lending further support to the concept that this mucosa represents a nonspecific reactive change.

In our study, one half of the cases with HPC had this alteration in mucosa immediately adjacent to the adenocarcinoma. However, in two of these cases with adjacent HPC, there was no morphologic evidence of traditional dysplasia adjacent to the carcinoma. In addition, in 7 of 10 cases with HPC, this change was found in mucosa distant from the carcinoma. In the latter cases, it is difficult to attribute this change as transitional mucosa. These areas did not seem to be associated with nonneoplastic mass lesions or strictures but rather arose in areas that otherwise resembled typical Crohn's disease.

To the authors' knowledge, there is only one published study that has evaluated p53 immunohistochemical expression in transitional mucosa. Using frozen tissue samples, Connelly et al. (23) analyzed p53 protein expression in transitional mucosa, normal mucosa, and tumor from 20 colorectal adenocarcinomas. In all 20 cases, the transitional and normal mucosae failed to express p53, whereas $65 \%$ of the adenocarcinomas showed intense nuclear immunoreactivity. On the basis of this data, the authors concluded that the absence of p53 staining in transitional mucosa did not support the preneoplastic nature of this mucosa.

In our study, one half of the cases of Crohn'srelated adenocarcinoma with HPC showed strong nuclear immunoreactivity for p53, with more intense staining at the base of the mucosa than on the surface. The p53-positive cases included HPC foci both distant from and adjacent to adenocarcinoma. In fact, one of two cases with HPC adjacent to adenocarcinoma without recognizable adjacent dysplasia stained for p53. In contrast, none of the HPC foci in cases of Crohn's disease without adenocarcinoma stained for p53.

In conclusion, HPC is found significantly more commonly in mucosa both adjacent to and distant from Crohn's-related adenocarcinoma when compared with age- and site-matched control specimens. In addition, p53 immunoreactivity is more commonly seen in HPC in cases of Crohn's-related adenocarcinoma compared with controls. These data suggest that this mucosal alteration may, in some cases, represent an unusual form of dysplasia in this setting.

\section{REFERENCES}

1. Choi PM, Zelig MP. Similarity of colorectal cancer in Crohn's disease and ulcerative colitis: implications for carcinogenesis and prevention. Gut 1994;35:950-4.

2. Connell WR, Sheffield JP, Kamm MA, Ritchie JK, Hawley PR, Lennard-Jones JE. Lower gastrointestinal malignancy in Crohn's disease. Gut 1994;35:347-52.

3. Hamilton SR. Colorectal carcinoma in patients with Crohn's disease. Gastroenterology 1985;89:398-407.

4. Ekbom A, Helmick C, Zack M, Adami H-O. Ulcerative colitis and colorectal cancer: a population-based study. N Engl J Med 1990;323:1228-33.

5. Harpaz N, Talbot IC. Colorectal cancer in idiopathic inflammatory bowel disease. Semin Diagn Pathol 1996;13:339-57.

6. Sigel JE, Petras RE, Lashner BA, Fazio VW, Goldblum JR. Intestinal adenocarcinoma in Crohn's disease: a report of 30 cases with a focus on coexisting dysplasia. Am J Surg Pathol 1999;23:651-5.

7. Filipe MI. Value of histochemical reactions for mucosubstances in the diagnosis of certain pathological conditions of the colon and rectum. Gut 1969;10:577-86.

8. Dawson PA, Filipe MI. An ultrastructural and histochemical study of the mucous membrane adjacent to and remote from carcinoma of the colon. Cancer 1976;37:2388-98.

9. Saffos RO, Rhatigan RM. Benign (nonpolypoid) mucosal changes adjacent to carcinomas of the colon: a light microscopic study of 20 cases. Hum Pathol 1977;8:441-9.

10. Filipe MI. The value of a study of the mucosubstances in rectal biopsies from patients with carcinoma of the rectum and lower sigmoid in the diagnosis of premalignant mucosa. J Clin Pathol 1972;25:123-8.

11. Lev R, Grover R. Precursors of human colon cancer: a serial section study of colectomy specimens. Cancer 1981;47:200715.

12. Robey-Cafferty SS, Ro JY, Ordonez NG, Cleary KR. Transitional mucosa of colon. A morphological, histochemical and immunohistochemical study. Arch Pathol Lab Med 1990; 114:72-5.

13. Isaacson P, Attwood PRA. Failure to demonstrate specificity of morphological and histochemical changes in mucosa adjacent to colonic carcinoma (transitional mucosa). J Clin Pathol 1979;32:214-8.

14. Listinsky CM, Riddell RH. Patterns of mucin secretion in neoplastic and non-neoplastic diseases of the colon. Hum Pathol 1981;10:923-9.

15. Mori M, Shimono R, Adachi Y, Matsuda H, Kuwano H, Sugi- 
machi K, et al. Transitional mucosa in human colorectal lesions. Dis Colon Rectum 1990;33:498-501.

16. Sawady J, Friedman MI, Katzin WE, Mendelsohn G. Role of the transitional mucosa of the colon in differentiating primary adenocarcinoma from carcinomas metastatic to the colon. An immunohistochemical study. Am J Surg Pathol 1991;15:136-44.

17. Riddell RH, Levin B. Ultrastructure of the "transitional mucosa” adjacent to large bowel carcinoma. Cancer 1977;40: 2509-22.

18. Marcheggiano A, Iannoni C, Palline F, Fieri G, Gallucci M, Caprilli R. Abnormal patterns of colonic mucin secretion after uretero-sigmoidostomy. Hum Pathol 1984;15:647-50.

19. Sunter JP, Higgs MJ, Cowan WK. Mucosal abnormalities at the anastomosis site in patients who have had intestinal resection for colonic cancer. J Clin Pathol 1985;38: 385-9.

20. Franzin G, Scarpa A, Dina R, Novelli P. “Transitional” and hyperplastic-metaplastic mucosa occurring in solitary ulcer of the rectum. Histopathology 1981;5:527-33.

21. Rhatigan RM, Saffos RO. Mucosal hyperplasia in colonic diverticula. Histopathology 1979;3:153-60.

22. Langlois NEI, Park KGM, Keenan RA. Mucosal changes in the large bowel with endometriosis: a possible cause of misdiagnosis of colitis? Hum Pathol 1994;25:1030-4.

23. Connelly JH, Bruner JM, Robey-Cafferty SS, Sahin A. p53 protein expression in transitional mucosa and adenocarcinomas of the colorectum. Mod Pathol 1992;5:537-9.

\section{Book Review}

\section{Benjamini E, Coico R, Sunshine G: Immunol- ogy, 4th Ed, 498 pp, New York, Wiley-Liss, 2000 (\$48.50).}

Nonimmunologists who occasionally visit the overcrowded arena of immunology books, like this reviewer, probably remember the book of Dr. Benjamini and his associates by its motto "less is more." Coping with a deluge of new discoveries makes the task of living by this motto extremely hard, but here is the best that these experienced educators have come up-all condensed into 500 pages. I thought that a few details here and there could have been deleted without much harm, but then, I imagine the authors would have been criticized for not having included this or that "essential cytokine" or such a "major breakthrough" as the lectin activated pathway of complement activation. Overall, I think that they have completed their task marvelously.

For the fourth edition, the authors have revamped the book and included hundreds of color drawings. They have rewritten much of the text and added good review material for the students. There is no doubt that these changes have increased the didactic value of the book and made it even more attractive. I like especially the study material at the end of each chapter because it forces students to think and apply their newly acquired knowledge. Although this features seems to be aimed primarily at medical students, college undergraduates could use it as well.

Immunology teachers who are using the previous editions of this book will be pleased by the latest revision, and I suspect that some new adoptions will be forthcoming. The book is well suited for one-semester college courses of immunology. I do not believe that the medical students could read it in the usual time allotted to immunology in the new curricula, but it has good material that neither the students nor their professors should ignore.

\section{Ivan Damjanov}

University of Kansas School of Medicine

Kansas City, Kansas 